Revista de Psicología de la PUCP. Vol. XIV. No 2. 1996

\title{
DIFERENCIAS DE GENERO EN EL PATRÓN DE CONDUCTA TIPO A EN UN GRUPO DE ESTUDIANTES UNIVERSITARIOS DE LIMA METROPOLITANA*
}

\author{
Ramón León' y David Sirlopún \\ Universidad Ricardo Palma
}

El Inventario de Autoreporte de Conducta Tipo A de Blumenthal y cols., que consta de ítemes en forma de adjetivos, fue respondido por 1145 estudiantes universitarios de ambos géneros, cuyo promedio de edad era 20 años. Los resultados ofrecen una visión singular acerca de los estereotipos que las mujeres y los hombres se asignan a si mismos. Entre los hombres, los adjetivos guardan mayor concordancia con los estereotipos masculinos, mientras que entre las mujeres si bien predominan adjetivos femeninos, también aparecen otros masculinos, en contraposición con lo habitual en una sociedad como la peruana. La explicación más plausible es que se ha trabajado con una muestra de clase media. Las mujeres conservan algunos estereotipos femeninos, pero también asumen estereotipos masculinos. Palabras claves: personalidad, Tipo A, Blumenthal, estudiantes universitarios, género, estereotipos.

Gender differences of Type $A$ behavior in a group of university students in Lima

A Spanish version of the Type A Self-Rating Inventory by Blumenthal et al., which includes items in the form of adjectives, was answered by 1145 university students of both genders and whose mean age was 20 years. Results give a particular view concerning the stereotypes males and females have about themselves. Among males adjectives such as honest, sure and energetic predominate. Among females predominate sensible and sociable but also enthusiastic and enterprising, in opposition with the traditional role assigned to women in a society such as the Peruvian. The suggested explanation is that females keep some tradicional feminine stereotypes but at the same time, take male roles.

Keywords: personality, Type A, Blumenthal, university students, gender, stereotypes

* Los autores desean expresar su agradecimiento a la Fundación Konrad Adenauer (KonradAdenauer-Stiftung), de Bonn, en Alemania Federal, por el apoyo económico prestado a la preparación del presente reporte.

1 Universidad Ricardo Palma (Lima). e Instituto Nacional de Salud Mental "Honorio DelgadoHideyo Noguchi”. Al momento de la preparación del presente reporte (Enero-Febrero 1995) Profesor Visitante del Instituto de Psicología (Director: Prof. Dr. Wolfgang Mertens) de la Ludwig-Maximilians-Universität, de Munich (Alemania Federal).

2 Universidad Ricardo Palma (Lima, Perú) e Instituto Nacional de Salud Mental "Honorio Delgado-Hideyo Noguchi". 

Cuando M. Friedman \& R. H. Rosenman propusieron el concepto de Conducta Tipo A lo hicieron teniendo en mente un factor de riesgo para el desarrollo de trastornos cardiaco-coronarios. Con este constructo describían "disposiciones conductuales como ambición, agresividad, competitividad e impaciencia; comportamientos específicos como alerta, tensión muscular, estilo de habla rápido y enfático y reacciones emocionales como marcada irritabilidad y signos expresivos de cólera" (Rosenman \& Chesney, 1982; p. 549).

Una relación significativa entre el Patrón de Conducta Tipo A (PCTA) y las enfermedades coronarias fue propuesta por ellos (Friedman \& Rosenman, 1959; Rosenman \& Friedman, 1961). Aunque ya antes se habían publicado observaciones sobre la probable vinculación entre impaciencia, propensión a la ira y trastornos coronarios (Kemple, 1945; Menninger \& Menninger, 1936), fueron sus trabajos los que constituyeron el punto de partida para una incesante labor de investigación. Uno de los estudios más ambiciosos fue The Western Collaborative Group Study (WCGS), investigación de corte prospectivo conducida en los Estados Unidos de Norteamérica durante ocho años y medio (en 3154 varones, entre 39 y 59 años de edad), cuyos resultados (Rosenman et al., 1975; 1976) influyeron para que el PCTA fuera designado en 1981 como un factor de riesgo para trastornos cardiacos por un panel auspiciado por el National Heart, Lung and Blood Institute (NHLBI) (Review Panel, 1981). Con esta designación, señalan Dembroski \& Czajkowski (1989), "por primera vez una variable psicosocial fue aceptada por la comunidad médica como factor de riesgo para los trastornos cardiaco-coronarios" (p. 25).

Si bien el PCTA continúa vinculado con dichos trastornos, es cada vez mayor la polémica acerca de la real naturaleza y alcance de dicho vínculo. Tras tantos años de investigación y de discusión, de categóricas 
afirmaciones así como de planteamientos antagónicos, se puede decir que en la actualidad se ha relativizado cada vez más el valor predictivo del concepto en sí y emprendido una activa labor de investigación en lo concerniente a variables mediadoras, entre las cuales la hostilidad ocupa el primer lugar (Adler \& Matthews, 1994; Smith \& Pope, 1991).

Hay inclusive "terceras posiciones": por ejemplo, aquella que plantea que el PCTA puede desarrollarse como una adaptación a una propensión constitucional (genéticamente transmitida) a la hiperactividad fisiológica mediada por el sistema nervioso simpático. De ser así, "el PCTA y los trastornos cardiaco-coronarios serían expresiones diferentes de la misma vulnerabilidad transmitida genéticamente y no podría asignarse ningún rol contributor al PCTA en los mencionados trastornos" (Holroyd \& Coyne, 1987; p. 68).

La discusión ha traído como una consecuencia adicional que el PCTA haya sido estudiado desde las más diversas perspectivas. Así, se han investigado los efectos de las hormonas en la conducta tipo A (Berman et al, 1993); la agresión hostil y su vinculación con ella (Check \& Dick,1986); la expresión de la agresión interpersonal en personas con conducta tipo A y tipo B (Holmes \& Will, 1985; Strube et al, 1984); la elevación de respuestas fisiológicas y endocrinas en el enfrentamiento de individuos tipo A a tareas cognitivas (Williams et al., 1982); la autoevaluación y la conducta tipo A (Henley \& Furnham, 1989); la conducta tipo A y la ética protestante de trabajo (Furnham, 1990); y, por supuesto, las relaciones entre el PCTA y la salud en general (Shoham-Yakubovich et al, 1988; para visiones panorámicas véase además Adler \& Matthews, 1994, esp. pp. 238-239).

Dada la diversidad de temas abordados con respecto al PCTA durante las tres últimas décadas resulta más bien sorprendente que las diferencias sexuales hayan sido objeto de muy pocos trabajos. Dichas diferencias han sido ( $y$ continúan siendo) uno de los filones más ricos de la investigación psicológica pues, como afirma Leona Tyler (1972), “a ninguna materia se le ha concedido un interés más permanente que a la de las diferencias entre los sexos" (p. 241).

Pero, como decimos, esto no se aplica plenamente al estudio del PCTA. Por el contrario, éste se ha concentrado especialmente en adultos 
del sexo masculino (Thoresen \& Law, 1991) y tan sólo en los últimos años se han reportado investigaciones con mujeres (Baker et al., 1984). Thoresen \& Law (1991) proponen una explicación para el carácter unilateral de la investigación del PCTA: "el constructo de Conducta Tipo A emergió originalmente como un modelo masculino por una buena razón, desde que el aumento de la morbilidad y mortalidad de trastornos cardiaco-coronarios en los años 50 y 60 afectó primariamente a hombres. El concepto de conducta tipo A se desarrolló en gran medida a partir de las observaciones clínicas y frecuentemente cualitativas de pacientes coronarios masculinos. Como se anotó, carecemos de observaciones similares en mujeres. Para decirlo de modo simple, no tenemos al presente una comprensión muy clara, ni científica ni clínica, de la Conducta Tipo A en mujeres" (p. 118).

La concentración del estudio del PCTA en hombres ha generado un problema adicional de carácter técnico, referido al diseño de los instrumentos de evaluación. El reactivo que suele ser considerado como el más preciso y seguro en la evaluación del PCTA, el Type A Standard Interview (Booth-Kewley \& Friedman, 1987), plantea algunas dificultades cuando se lo emplea con mujeres. "Muchas de las preguntas del SI están orientadas hacia el trabajo y la conducta competitiva, que pueden ser socialmente aceptables para hombres que trabajan, pero no para mujeres, en especial para amas de casa", escriben Matthews \& Haynes (1986).

La tabla 1 presenta algunos de los rasgos típicos de la conducta tipo A, tal como han sido mencionados en la literatura especializada.

La competitividad, la agresividad y la ambición se encuentran entre los rasgos de la conducta Tipo A frecuentemente mencionados en la literatura psicológica entre 1959 y 1979. Aun cuando, por un lado, ellos son categorías sociales (Maccoby, 1988) y por lo tanto son pasibles de cambios con el paso del tiempo y la evolución social; y del otro, hay presunciones de que entre hombres y mujeres no existen en realidad tantas diferencias (Frieze et al, 1978), lo cierto es que dichos rasgos forman parte del estereotipo del sexo masculino.

La relación de rasgos de conducta presentada en la Tabla 1 permite, asimismo, reconocer cuántas características han sido involucradas en el PCTA. No todas deben ser consideradas como negativas o con efectos dañinos para la salud. Es así que mientras algunas (como la hostilidad), 
Tabla 1: Rasgos de la conducta Tipo A seńalados en la literatura entre 1959-1979

\begin{tabular}{|c|c|c|c|}
\hline \multirow[t]{2}{*}{ Rasgo } & \multirow[b]{2}{*}{ Total } & \multicolumn{2}{|c|}{ Frecuencia de citación } \\
\hline & & $1959-1974$ & $1975-1979$ \\
\hline Competitividad & 72 & 27 & 45 \\
\hline Prisa & 62 & 24 & 38 \\
\hline Agresividad & 44 & 13 & 31 \\
\hline Drive & 41 & 22 & 19 \\
\hline Búsqueda de logros & 33 & 09 & 24 \\
\hline \multirow{2}{*}{\multicolumn{2}{|c|}{ Ambición, deseos }} & 14 & 14 \\
\hline & 26 & 14 & 12 \\
\hline Ritmo acelerado & 25 & 12 & 13 \\
\hline Impaciencia & 24 & 09 & 15 \\
\hline Hostilidad & 19 & 02 & 17 \\
\hline Manierismos motores & 18 & 11 & 07 \\
\hline Hiperalerta & 14 & 04 & 10 \\
\hline Manierismos del habla & 13 & 06 & 07 \\
\hline Esfuerzo & 13 & 06 & 07 \\
\hline Manejo duro de situac. & 12 & 05 & 07 \\
\hline Ausencia de descanso & 12 & 08 & 04 \\
\hline Compromiso con trabajo & 12 & 05 & 07 \\
\hline \multicolumn{4}{|l|}{$\begin{array}{l}\text { Involucración en } \\
\text { demasiadas cosas }\end{array}$} \\
\hline \multicolumn{4}{|l|}{ Extremadamente } \\
\hline conciente/responsable & 11 & 06 & 05 \\
\hline Búsqueda de & 10 & 08 & 02 \\
\hline \multicolumn{4}{|l|}{$\begin{array}{l}\text { Estilo de confrontación } \\
\text { destinado a controlar }\end{array}$} \\
\hline los acontecimientos & 07 & 00 & 07 \\
\hline Exito en el trabajo & 03 & 03 & 00 \\
\hline Alta productividad & 03 & 01 & 02 \\
\hline Alto nivel de actividad & 03 & 02 & 01 \\
\hline \\
\hline \multirow{2}{*}{\multicolumn{4}{|c|}{$\begin{array}{l}\text { Ausencia o pocas } \\
\text { actividades no-laborales }\end{array}$}} \\
\hline & 02 & 01 & 01 \\
\hline \multicolumn{4}{|l|}{ Perfeccionismo, } \\
\hline altos estándares & 01 & 01 & 00 \\
\hline Ansiedad & 01 & 01 & \\
\hline \multicolumn{4}{|l|}{ Incapacidad para } \\
\hline definir límites & 01 & 00 & 00 \\
\hline Expresividad & 01 & 00 & 00 \\
\hline Necesidad de poder & 01 & 00 & 01 \\
\hline \multicolumn{4}{|l|}{ Número de artículos } \\
\hline revisados & 101 & 44 & 57 \\
\hline
\end{tabular}

Tomado de Furnham (1990) 
tienen una naturaleza "tóxica", otras como el manejo duro de ciertas situaciones y la búsqueda de logros no tienen una importancia decisiva en el desarrollo de trastornos cardiaco-coronarios (Dembroski \& Czajkowski, 1989) y, más bien, pueden inclusive ser promocionadoras del avance laboral de quienes las presenten (Consoli \& Antonelli, 1992).

Lo dicho en el párrafo previo nos lleva a considerar los rasgos del PCTA, sin perjuicio de su significado para el terreno de la salud, también como conductas influidas y determinadas por procesos de socialización y expectativas y normas que rigen una sociedad determinada en una época determinada. Como tales, la exploración de dichos rasgos así como la del grado en el cual las mujeres y los hombres creen poseerlos (que es lo que en el presente trabajo se reporta) tornan posible acceder a información que permita una visión cada vez más diferenciada acerca de la psicología del género en el Perú.

Hombres y mujeres están sometidos en el Perú a procesos de socialización muy diferentes aún hoy, a menos de dos mil días del año 2000 . La bibliografía sobre el particular es abundante y no puede ser tratada en detalle acá, pero ella pone en evidencia la presencia de rasgos de mayor asertividad, sociabilidad, liberalismo en el plano de las actitudes y de la conducta sexuales en los hombres que en las mujeres. De los hombres se espera agresividad, capacidad para imponerse, temprana iniciación sexual y alta sociabilidad; $y$ puede afirmarse que para todo ello son educados. Las mujeres, por el contrario, continúan siendo educadas para ser dóciles, sumisas, recatadas en el plano sexual y con una sociabilidad que no asuma las formas expansivas que por lo general tiene la de los hombres (García Trovato, 1992). Esto es, se promociona (y se espera) en los hombres "conductas masculinas" y en las mujeres "conductas femeninas" (Ragúz de Romaña, 1988).

Así, por ejemplo, "las mujeres en particular, se inhiben al conversar sobre aspectos íntimos de su vida, especialmente por el énfasis cultural y religioso que tiene la virginidad en el Perú, mientras que la información que dan los varones, en cuanto al nivel y frecuencia de su actividad sexual, es generalmente exagerada porque, al parecer, quieren asentar su machismo" (Ferrando et al., 1989; pg. 39). Pero esto en modo alguno se limita al terreno de las actitudes y conductas sexuales. Jeanine Anderson encontró 
en su obsevación en colegios, que "la estrategia varonil para lograr que la profesora les prestara atención era portarse mal; la correspondiente estrategia de las mujercitas era el conformismo exacto a las normas y expecrativas" (1987; p. 128), mientras que León y Portocarrero (1989) hallaron en una investigación sobre autoconciencia en una zona deprivada de Lima niveles de ansiedad social más elevados entre las mujeres. Una amplia revisión de hallazgos de la investigación psicológica sobre el particular en tesis universitarias se encuentra en Lazo Manrique (1988).

Estos rasgos diferenciales en el proceso de socialización se evidencian en todas las clases sociales de nuestro país, si bien asumen manifestaciones de mayor dramatismo en las menos favorecidas.

Un trabajo previo acerca de diferencias sexuales en los rasgos del PCTA en adolescentes peruanos fue conducido por León \& Romero en 1988. Los autores llevaron a cabo un estudio con adolescentes que cursaban la instrucción secundaria en una zona urbano-marginal de Lima. Trescientos cuatro adolescentes (135 hombres y 169 mujeres) respondieron la versión castellana del Type A-Type B Questionnaire, de Eysenck \& Fulker (1983), un reactivo que evalúa cuatro factores: tensión, ambición, actividad y ausencia de represión. El Gráfico 1 informa acerca de los resultados de dicho estudio.

En líneas generales, los resultados de aquel trabajo concordaban con la idea que se tiene acerca de la conducta de los hombres: éstos obtuvieron promedios más elevados que las mujeres en ambición, actividad y ausencia de represión. Sin embargo, las mujeres superaron a los hombres en tensión.

De los catorce ítemes que evalúan tensión en el Type A-Type B Questionnaire, las mujeres puntuaron más alto que los varones en doce. Ellas negaron ser "conversadoras, calmadas y tranquilas" (item 10) y afirmaron "amargarse fácilmente" más que los hombres (1.98 versus 1.62). En los ítemes de todas las otras escalas, con excepción de dos casos (ítemes 5 y 9 " ¿Necesita Ud. de constante reconocimiento para avanzar en el trabajo?"; $y$, “ $¡$ es Ud. conciente de las presiones del tiempo, y de citas y acuerdos?", respectivamente), fueron los hombres los que obtuvieron promedios más elevados. 
Gráfico 1: Promedios de hombres y mujeres en las 4 áreas del Type A Type B. Cuestionario de Eysenck \& Fulker, en el estudio de León \& Romero (1988)
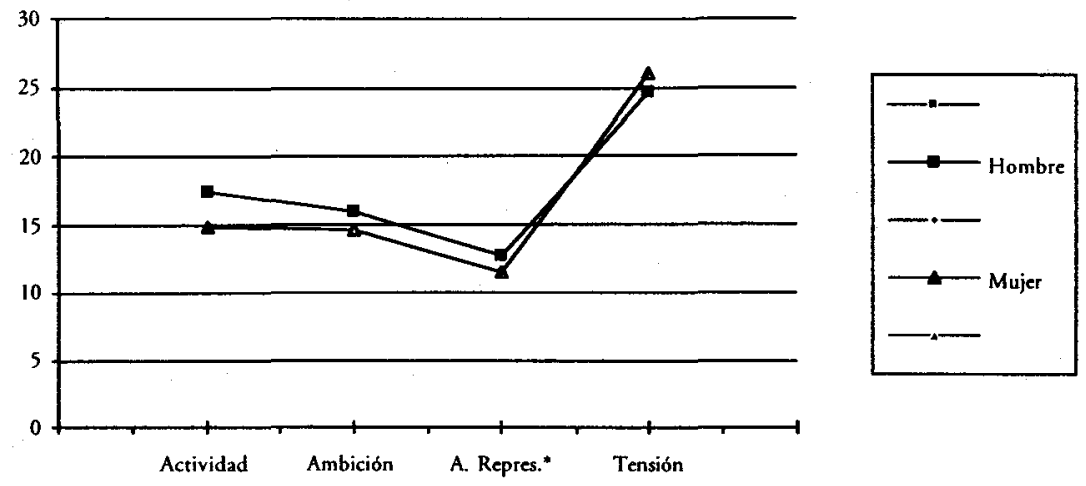

Es necesario, sin embargo, hacer una acotación. El trabajo de León \& Romero (1988) se llevó a cabo con adolescentes de una zona deprivada de Lima, caracterizada por su extrema pobreza: Villa María del Triunfo. En su estudio acerca de la desigualdad económica en el Perú, Amat y León (1986) ubica a Villa María del Triunfo en el Estrato Cinco, que comprende a zonas de los niveles más bajos en materia de calidad de vida, indicando que "los pobladores de estos distritos llevan una vida de seis a doce veces inferior a los del primer estrato" (p. 23), en el que ubica a San Isidro, La Punta, Jesús María y Miraflores.

En una zona como ésta, el proceso de socialización puede asumir formas de particular dureza y llegar a tener características traumáticas. En un trabajo acerca de prácticas de crianza en madres de estratos socioeconómicos medio y bajo de Lima, Majluf (1989) seńala que "los padres de la clase menos favorecida generalmente son más autoritarios, restrictivos, tienen poca comunicación con sus hijos, enfatizan la obediencia, el respeto, el evitar problemas y usan más el castigo físico" (p. 154).

No sorprende pues que, por ejemplo, Perales et al. (1985) trabajando en otro distrito de Lima ubicado en el Estrato Cinco, Independencia, encontraran que "un buen número de hijos reporta manifestaciones de 
irritabilidad, tensión y dificultades en el sueño que podrían sugerir indicadores de estrés o manifestaciones iniciales de un síndorme ansiosodepresivo" (p. 66).

La situación es algo diferente en la clase media. En el trabajo antes mencionado, Majluf (1989) indica que "los estudios comparativos entre familias de clase socio-económica media y baja han revelado diferencias consistentes en las actitudes y valores de éstos, observándose que los primeros tienden a ser más democráticos, permisivos, afectuosos, comunicativos, estimulan más la independencia, el control de sí mismos y la creatividad de sus hijos" (p. 154).

Dado el carácter genérico del concepto de clase media, es necesario precisar matices con respecto a la afirmación de Majluf. Eso es lo que hace Ragúz de Romaña al escribir lo siguiente: "en Lima se han hecho estudios de corte sociológico con mujeres de clase media ..., evidenciando su conservadurismo, por ejemplo, privilegiando la educación del hijo hombre y creyendo en una tendencia "innata" de la mujer a entregarse a sus hijos" (Ragúz de Romaña, 1988; p. 96).

Las diferencias en el proceso de socialización del infante vinculadas a la clase social provienen, a su vez, de los procesos de socialización de sus progenitores. "Cuando nace un niño, su madre, por un simple vistazo a los genitales, se informa con respecto a su sexo", y, "luego, de acuerdo con su propia experiencia en la vida -una confluencia de actitudes derivadas de la sociedad, de sus padres, de sus propias luchas por crear su identidad del género y de su interacción con el padre del bebé- moldea el género de su infante", escribe Stoller (1981; p. 275).

Este proceso de socialización diferencial debe teóricamente dar lugar a diferentes constelaciones de rasgos de personalidad en adolescentes de clase media y de clase baja dado que, como lo señala McClelland (1981), desde los años treinta (sobre todo debido al trabajo de Linton y Kardiner) el desarrollo de la personalidad es visto como el producto de condiciones ambientales y de tempranas experiencias infantiles.

Las dos preguntas que llevaron a realizar el presente trabajo fueron: (1) ¿qué características asume el PCTA en jóvenes y adolescentes de clase 
media?; y, (2) ¿cómo se perciben ellos a sí mismos en referencia a una serie de rasgos de conducta propios del PCTA?.

\section{Metodología}

Mil ciento setenta y ocho estudiantes de una universidad particular respondieron el Inventario de Autoreporte de Conducta Tipo A de Blumenthal et al. (1985). La universidad está ubicada en la zona sur de Lima y tiene alrededor de 10,000 estudiantes, los que en líneas generales provienen de hogares de clase media.

Por diferentes razones, 33 pruebas fueron descartadas, de modo tal que la muestra final fue de 1145 alumnos ( 639 mujeres con una edad promedio de 20.4 ańos, DS 2.63; y, 506 hombres, 21.3, DS 2.89).

El Inventario de Autoreporte de Conducta Tipo A de Blumenthal et al. consta de 38 ítemes, de los cuales 28 evalúan la Conducta Tipo A. Cada item presenta un adjetivo ("agresivo", "enérgico", "dinámico", "testarudo") a ser calificado en una escala de 1 ("nunca o casi nunca es verdad") a 7 ("siempre o casi siempre es verdad"). La versión castellana del Inventario ha sido adaptada y validada en un trabajo previo (León et al., 1995).

Más allá de su utilidad como reactivo de medición del PCTA, el Inventario es apropiado también para la evaluación de la autopercepción. Los adjetivos que incluye designan características de la conducta que reciben (según el caso) aprobación o desaprobación social y que, asimismo, muchas veces suelen ser consideradas como "propias" de un sexo o del otro. Los ítemes pueden sin problemas ser aplicados tanto a hombres como a mujeres y, en el caso de la versión castellana, los autores colocaron el adjetivo tanto en femenino como en masculino.

\section{Resultados}

La Tabla 2 presenta los promedios obtenidos por hombres y mujeres en el presente estudio.

Como puede verse, las mujeres puntúan ligeramente superior a los hombres pero las diferencias no son grandes y no alcanzan el nivel de significación estadística. 
Tabla 2: Promedios y desviaciones standard de hombres y mujeres en el Inventario de Autoreporte de Blumenthal et al.

\begin{tabular}{lcc}
\hline & $\mathrm{X}$ & $\mathrm{DS}$ \\
\hline Hombres & 114.83 & 15.32 \\
Mujeres & 116.48 & 16.10 \\
\hline
\end{tabular}

En la Tabla 3 están los promedios de hombres y mujeres para cada uno de los ítemes. Puede observarse que por lo general las diferencias entre los promedios de hombres y mujeres no son muy grandes, pero algunas de ellas alcanzan significación estadística.

Con el propósito de determinar cuál era el orden de prioridad de los adjetivos para cada uno de los sexos se procedió a ubicarlos de acuerdo con su promedio. La Tabla 4 presenta los ítemes debidamente ordenados.

El adjetivo que tiene el promedio más alto entre las mujeres es "sensible" (5.89) mientras que "franco" es el que lo tiene entre los hombres (5.45); a su vez, éste ocupa el segundo lugar entre las mujeres (5.75). El segundo lugar entre los hombres corresponde a "seguro" (5.18). Resulta de interés observar que los diez primeros puestos son ocupados por los mismos adjetivos en ambos sexos (excepto para "hablador", sétimo lugar entre las mujeres pero décimotercero entre los hombres; y para "rápido", décimo entre los hombres y décimoprimero entre las mujeres).

$\mathrm{El}$ orden de los tres primeros adjetivos en los varones coresponde al estereotipo que se tiene de ellos: "franco", "seguro", "vigoroso" ("seguro" y "vigoroso" ocupan el sexto y octavo lugar entre las mujeres). El primero y el tercero, además, son por lo general considerados como característicos de la conducta tipo A (anótese también que otro rasgo descriptor del PCTA, "ambicioso", ocupa el sétimo lugar entre los hombres, pero el décimo entre las mujeres). El adjetivo "sensible" ocupa entre los hombres el quinto lugar y los promedios obtenidos por éstos y por las mujeres difieren notablemente (5.01 versus 5.89).

Los siete adjetivos restantes de los diez primeros en hombres y mujeres, cuando se les compara, no ofrecen mayores diferencias, con la única 
Tabla 3: Promedios y desviación standard para hombres y mujeres en los adjetivos del Inventario de Autoreporte de Conducta Tipo A de Blumenthal et al.

\begin{tabular}{|c|c|c|c|c|c|c|}
\hline & \multicolumn{2}{|c|}{ Femenino } & \multicolumn{2}{|c|}{ Masculino } & \multirow[t]{2}{*}{$\mathrm{F}$} & $P$ \\
\hline & $\mathrm{X}$ & DS & $\mathrm{X}$ & DS & & \\
\hline Dinámico(a) & 4.69 & 1.37 & 4.77 & 1.95 & 0.778 & 0.618 \\
\hline Tranquilo(a) & 3.42 & 1.60 & 3.44 & 1.61 & 0.043 & 0.830 \\
\hline Franco(a) & 5.75 & 1.13 & 5.45 & 1.35 & 16.382 & $0.0001 *$ \\
\hline Seguro(a) & 4.92 & 1.49 & 5.18 & 1.42 & 8.888 & $0.003 *$ \\
\hline Pacífico (a) & 2.80 & 1.48 & 2.71 & 1.50 & 1.073 & 0.300 \\
\hline Agresivo(a) & 2.82 & 1.54 & 2.72 & 1.42 & 1.322 & 0.248 \\
\hline Rápido(a) & 4.54 & 1.37 & 4.76 & 1.33 & 7.351 & 0.006 \\
\hline Calmado(a) & 3.43 & 1.49 & 3.28 & 1.50 & 2.730 & 0.094 \\
\hline Enérgico(a) & 4.36 & 1.51 & 4.49 & 1.43 & 1.877 & 0.167 \\
\hline Emprendedor(a) & 5.06 & 1.31 & 4.81 & 1.36 & 10.100 & $0.001 *$ \\
\hline Sosegado(a) & 4.26 & 1.52 & 4.25 & 1.45 & 0.010 & 0.918 \\
\hline Testarudo(a) & 3.74 & 1.72 & 3.62 & 1.65 & 1.312 & 0.250 \\
\hline Tenso(a) & 4.13 & 1.63 & 3.91 & 1.62 & 5.445 & $0.018^{*}$ \\
\hline Entusiasta & 5.31 & 1.20 & 5.02 & 1.30 & 15.873 & $0.000229^{*}$ \\
\hline Irritable & 3.64 & 1.65 & 3.67 & 1.68 & 0.106 & 0.743 \\
\hline Ambicioso(a) & 4.57 & 1.75 & 4.83 & 1.76 & 6.157 & $0.012 *$ \\
\hline Dominante & 4.12 & 1.86 & 4.36 & 1.67 & 4.941 & $0.024 *$ \\
\hline Asertivo(a) & 4.39 & 1.40 & 4.28 & 1.42 & 1.650 & 0.196 \\
\hline Discutidor(a) & 4.33 & 1.63 & 4.62 & 1.64 & 8.491 & $0.003^{*}$ \\
\hline Sensible & 5.89 & 1.33 & 5.01 & 1.63 & 10.671 & $0.0000^{*}$ \\
\hline Sereno(a) & 3.57 & 1.51 & 3.29 & 1.46 & 10.125 & $0.001 *$ \\
\hline Bullero(a) & 3.31 & 1.77 & 3.43 & 1.76 & 1.301 & 0.252 \\
\hline Egoísta & 2.67 & 1.48 & 2.71 & 1.45 & 0.211 & 0.651 \\
\hline Buen carácter & 2.86 & 1.37 & 2.83 & 1.42 & 0.089 & 0.763 \\
\hline Hablador(a) & 4.89 & 1.61 & 4.41 & 1.62 & 24.033 & $0.000025 *$ \\
\hline Sociable & 5.26 & 1.40 & 4.91 & 1.54 & 16.753 & $0.001 *$ \\
\hline Precavido(a) & 2.75 & 1.46 & 2.82 & 1.43 & 0.589 & 0.550 \\
\hline Vigoroso(a) & 4.86 & 1.39 & 5.15 & 1.37 & 12.077 & $0.0008 *$ \\
\hline
\end{tabular}

* $<0.05$ [gl: $1 / 1143$ ] 
Tabla 4: Orden de los adjetivos por sexo con indicación del promedio

\begin{tabular}{lll}
\hline $\mathrm{N}^{\circ}$ & \multicolumn{1}{c}{ Mujeres } & \multicolumn{1}{c}{ Hombres } \\
\hline 01 & Sensible (5.89) & Franco (5.45) \\
02 & Franco (5.75) & Seguro (5.18) \\
03 & Entusiasta (5.31) & Vigoroso (5.15) \\
04 & Sociable (5.26) & Entusiasta (5.02) \\
05 & Emprendedor (5.06) & Sensible (5.01) \\
06 & Seguro (4.92) & Sociable (4.91) \\
07 & Hablador (4.89) & Ambicioso (4.83) \\
08 & Vigoroso (4.86) & Emprendedor (4.81) \\
09 & Dinámico (4.69) & Dinámico (4.77) \\
10 & Ambicioso (4.57) & Rápido (4.76) \\
11 & Rápido (4.54) & Discutidor (4.62) \\
12 & Asertivo (4.39) & Enérgico (4.49) \\
13 & Enérgico (4.36) & Hablador (4.41) \\
14 & Discutidor (4.33) & Dominante (4.36) \\
15 & Sosegado (4.26) & Asertivo (4.28) \\
16 & Tenso (4.13) & Sosegado (4.25) \\
17 & Dominante (4.12) & Tenso (3.91) \\
18 & Testarudo (3.74) & Irritable (3.62) \\
19 & Irritable (3.64) & Testarudo (3.62) \\
20 & Sereno (3.57) & Tranquilo (3.44) \\
21 & Calmado (3.49) & Bullero (3.43) \\
22 & Tranquilo (3.42) & Sereno (3.29) \\
23 & Bullero (3.31) & Calmado (3.28) \\
24 & De buen carácter (2.86) & De buen carácter (2.83) \\
25 & Agresivo (2.82) & Precavido (2.82) \\
26 & Pacífico (2.80) & Agresivo (2.76) \\
27 & Precavido (2.75) & Egoísta (2.71) \\
28 & Egoísta (2.67) & Pacífico (2.71) \\
\hline & &
\end{tabular}


excepción de "hablador". Las mujeres lo colocan en el sétimo lugar, dos puestos por debajo de "sociable", pero los hombres lo ubican en el décimotercer lugar ("sociable", sin embargo, ocupa el sexto lugar en ellos).

Tan interesante como el examen de los diez primeros adjetivos es el de los siguientes. Los hombres -en comparación con las mujeres- se ven como más discutidores, enérgicos, dominantes y ligeramente más irritables, pero éstas son más asertivas, serenas y testarudas. Algo llama, sin embargo, la atención: las mujeres obtienen un promedio mayor que los hombres (4.13 versus 3.91) en el adjetivo "tenso", una suerte de confirmación de lo que hallaron León \& Romero (1988).

Los últimos ocho adjetivos merecen asimismo un breve comentario, especialmente porque algunos hallazgos parecen contradictorios. Así, los hombres se ven al mismo tiempo como más tranquilos y bulleros que las mujeres, pero menos pacíficos que ellas. Las mujeres a su vez se ven como más agresivas (2.82 versus 2.76$)$. Sorprende encontrar entre los últimos íremes adjetivos como "de buen carácter", ubicado tanto en hombres como en mujeres en el número 24. Algo semejante puede decirse de "pacífico" (26. en las mujeres, y 28. en los hombres). Cabe preguntarse qué connotaciones pueden haber tenido estos dos adjetivos entre los sujetos con los cuales se llevó a cabo el presente estudio. Una posibilidad a considerar sería que "pacífico" halla sido entendido como descriptor de la tendencia a evadir retos y a una extrema pasividad, lo cual no es socialmente bien visto. En el caso de "agresivo", tal vez el término se entendió desde su lado negativo (propensión a la violencia) y no como indicador de firmeza y de seguridad así como de iniciativa en el actuar.

En líneas generales, la impresión que se obtiene del examen de la Tabla 3 es que la ubicación de los adjetivos en los hombres guarda mayor concordancia con la imagen habitual que se tiene de ellos, mientras que no se puede decir lo mismo en el caso de las mujeres. Ellos deben ser "seguros" (2.), "sociables" (6.), "emprendedores" (8), "dinámicos" (9.), "rápidos" (10.), "vigorosos" (3.), "francos" (1.). En las mujeres la imagen es menos concordante: ellas se ven al mismo tiempo como "sensibles" (1.), "vigorosas" (8.), "dinámicas" (9.) y "ambiciosas" (10.). 
Como parte del estudio de validación y confiabilidad del reactivo de Blumenthal que los autores llevaron a cabo (León et al., 1995), se procedió a realizar un análisis factorial. Por medio de él se identificaron 6 factores, que explican aproximadamente el $50 \%$ de la varianza. Dichos factores son: (I) Conducta competitiva (9 itemes); (II) Agresividad (5 itemes); (III) Gregarismo (5 ítemes); (IV) PasividadiActividad (5 ítemes); (V) Egocentrismo (3 ítemes); y, (VI) Tensión (2 ítemes) (véase León \& Sirlopú, 1995).

Para cada uno de estos factores se calcularon promedios y desviación standard por sexo, que se presentan en la Tabla 5. Puede observarse que los hombres y mujeres obtienen puntajes semejantes o muy parecidos en Agresividad y en Conducta competitiva. En Pasividad/Actividad las mujeres obtienen un puntaje mayor, así como en Gregarismo y en Tensión. Los hombres, por su parte, obtienen el puntaje mayor en Egocentrismo.

Tabla 5: Promedios y desviación standard por sexo para cada uno de los 6 factores del Inventario de Autoreporte de Blumenthal et al.

\begin{tabular}{lcccccc}
\hline Factor & \multicolumn{2}{c}{$\mathrm{N}^{\circ}$} & \multicolumn{2}{c}{ Hombres } & \multicolumn{3}{c}{ Mujeres } \\
& itemes & $\mathrm{X}$ & $\mathrm{DS}$ & $\mathrm{X}$ & $\mathrm{DS}$ & Zcal \\
\hline I. Conducta competitiva & 9 & 46.75 & 7.00 & 46.68 & 6.99 & -0.16 \\
II Agresividad & 5 & 17.35 & 4.78 & 17.35 & 5.32 & -0.02 \\
III.Gregarismo & 5 & 15.60 & 3.79 & 16.33 & 3.79 & $3.25^{*}$ \\
IV. Pasividad/Actividad & 5 & 14.27 & 4.12 & 14.69 & 4.43 & 1.65 \\
V. Egocentrismo & 3 & 11.91 & 3.60 & 11.37 & 3.77 & $-2.45^{*}$ \\
VI. Tensión & 2 & 8.92 & 2.51 & 10.03 & 2.28 & $7.71^{*}$ \\
\hline
\end{tabular}

* $\mathrm{p}<0.05[\mathrm{Zt}=1.96]$

\section{Discusión}

Como sucede con la inmensa mayoría de las investigaciones (no sólo las psicológicas, por cierto), los resultados obtenidos en la presente ofrecen múltiples y en algunos casos paradójicas -casi contradictorias- posibilidades de discusión y de interpretación. 
Esto se hace evidente en el hallazgo más importante de nuestro estudio, el que se da a conocer en la Tabla 1: que las mujeres del grupo con el que hemos trabajado presentan en promedio un puntaje mayor en conducta Tipo A que los varones. El hallazgo, creemos, moverá a sorpresa pues el PCTA está más referido a personas del sexo masculino (como ya lo dijimos), que son asimismo aquéllas en las cuales más se lo ha investigado.

La sorpresa puede aún ser mayor si se tiene en cuenta que el PCTA supone un complejo de afectos y conductas que, en una sociedad con una fuerte ideología machista como la nuestra, se espera encontrar sobre todo en varones y no en mujeres. Así es: prevalece todavía en el Perú una ideología patriarcal que estimula la toma de decisiones por parte del varón y el confinamiento de la mujer a la función doméstica, ideología que tiene tanta mayor fuerza cuanto más se desciende en la escala social (Chueca, 1985).

Sin embargo, en las conclusiones es necesaria cierta cautela. Cuando se revisa la Tabla 3 se constata que los adjetivos con los promedios más elevados son por lo general los mismos para un sexo y para el otro. Esto significa que, si bien las mujeres puntúan más alto que los hombres, no se observan diferencias substanciales en la selección de los adjetivos por parte de unas y otros.

Es precisamente la Tabla 3 la que ofrece la mayor cantidad de material para la discusión sobre el particular. La analizaremos en primer lugar en función de lo que podríamos llamar psicología de los géneros; o sea, como fuente de información acerca de diferencias por sexo, haciendo abstracción del PCTA, al que trataremos en segundo lugar.

El adjetivo "franco" es el que ocupa el primer lugar entre los hombres, mientras que es "sensible" el que lo hace entre las mujeres, algo que corresponde al estereotipo que se tiene de ellas (Frieze et al., 1978).

Cabe preguntarnos qué debemos entender por "sensible", pues el término tiene numerosas connotaciones. Una posibilidad es la de asociarlo a una elevada emocionalidad, que incluiría predisposiciones a la ansiedad, el temor y la hiperreactividad a estímulos ambientales (esto es, lo que Eysenck denomina neuroticismo). Si esta fuera la connotación correcta, 
la ubicación de "sensible" en primer lugar guardaría concordancia con hallazgos psicométricos en los que las mujeres puntúan más alto que los varones en algunas medidas de intensidad emocional (Banaji \& Prentice, 1994).

Pero hay aún otra alternativa, "sensible" puede entenderse más bien como indicador de "sensibilidad social", es decir interés por las otras personas, sus actividades, necesidades y estados de ánimo. Esto constituye asimismo, un rasgo frecuentemente considerado como femenino. También para esta segunda alternativa se encuentra respaldo en la literatura psicológica: Jose \& McCarthy (1988) utilizaron el Bem Sex Role Inventory y hallaron que las mujeres y sujetos con puntajes de alta femineidad fueron vistos como más preocupados acerca de los sentimientos de los miembros de un grupo.

El orden en el cual los adjetivos son ubicados por sexo corresponden, por supuesto, no a una realidad objetiva sino a autopercepciones, a estereotipos, los cuales no necesariamente guardan estricta comunión con los hechos.

Sin embargo, en algunos aspectos se tiene la impresión de que tal comunión se produce. Consideremos el caso del adjetivo "hablador", que se encuentra ubicado en el sétimo lugar entre las mujeres y en el décimotercero entre los varones. Maccoby \& Jacklin (1974) seńalaron que hay una diferencia de género a favor de los adolescentes de sexo femenino en habilidades verbales, mientras que los del sexo masculino destacaban más en habịlidades espaciales y cualitativas. Aunque esa afirmación ha sido relativizada por Feingold (1988), de hecho se suele ver a las mujeres como más "habladoras", más conversadoras y comunicativas que los hombres. En general, como más emotivas y, también, como más expresivas (Spence \& Helmreich, 1978).

No obstante, los resultados que reportamos deben -siguiendo un principio de elemental parsimonia científica- ser entendidos como la expresión de concepciones y estereotipos que determinan la imagen que se tiene de uno y otro sexo.

Hoy se sabe que el conocimiento de estos estereotipos se adquiere en una edad muy temprana (Best et al., 1977; Williams et al., 1975), pero 
también se tiene una clara visión de que el género -que "alude a las características particulares que culturalmente se atribuyen a cada uno de los sexos, es decir al contenido y a la definición social de las personas en función del sexo", de acuerdo con lo que señala Francke (1986; p. 309)es una variable de particular sensibilidad a los cambios sociales como, asimismo, a experiencias individuales de gran resonancia vivencial. Así, por ejemplo, Guzmán \& Portocarrero (1985) han estudiado en nuestro medio el significado de la experiencia migratoria y laboral en las mujeres.

A pesar de esto, sin embargo, algunos estereotipos (pero probablemente también algunas conductas, cuya presencia real sustenta los estereotipos) parecen resistir a las experiencias previamente descritas. Así, Ragúz de Romaña (1983) encontró que "los estereotipos más definidamente masculinos corresponden a autosuficiente, de fuerte personalidad, independiente, de empuje y ambicioso"; y, "los más definidamente femeninos corresponde a flexible y tierno" (p. 31). Resultados semejantes reportan en un reciente trabajo llevado a cabo en México Acuña et al. (1994) y algo de eso se puede verificar en los hallazgos del presente estudio. Los hombres ubican los adjetivos "ambicioso", "vigoroso", "seguro", “discutidor", y "enérgico" en posiciones superiores a las que las ubican las mujeres (Tabla 3). Ellos, asimismo, se ven como ligeramente más "competitivos" y más "egocéntricos", mientras que las mujeres obtienen promedios más altos en "gregarismo" y en "tensión".

Sin embargo, y a pesar del mayor promedio en tensión, las mujeres puntúan por encima de los hombres en pasividad, probablemente como consecuencia de la presión social en el sentido de que ellas se muestren más recatadas, menos audaces y emprendedoras en el plano de las relaciones sociales.

Los resultados ofrecen una visión un tanto abigarrada y, a nuestro parecer, en modo alguno uniforme. De un lado, es posible reconocer "correctamente ubicadas" a conductas y roles que por lo general se atribuyen a un sexo y al otro. Pero, de igual manera, se constata la presencia de similitudes llamativas, como por ejemplo la de que hombres y mujeres se ven a sí mismos como competitivos, si bien hay ligera predominancia de los hombres. 
La explicación más plausible para entender lo sucedido puede derivarse de las características sociológicas de la muestra con la cual hemos trabajado. Se trata, como ya se indicó, de una muestra universitaria; es decir, de personas que están siendo sometidas en esta etapa de su vida a un proceso de gran apertura de horizontes personales. La educación universitaria significa para hombres y mujeres posibilidades de competencia en las aulas, trato relativamente igualitario, prolongadas relaciones sociales con personas del sexo opuesto, estímulo de los intereses individuales. Si a esto se agrega la procedencia de hogares de clase media, con una atmósfera más estimuladora de la autoafirmación y de la independencia, podremos entender por qué hemos obtenido los resultados que aquí estamos reportando.

Esto es, mientras que los hombres universitarios se perciben (y se reafirman) en su rol masculino, las mujeres universitarias asumen en el plano de la autopercepción algunas de las características de ellos ("vigorosas", "dinámicas", "ambiciosas") conservando rasgos femeninos ("sensibles"). Los rasgos "no femeninos" que ellas asumen resultan a su vez necesarios para poder desenvolverse con mayor soltura en el medio laboral. Un "empleo adecuado", que supone un nivel de educación mayor (Barrig 1988), exige de ellas conductas de mucha afirmación; esto es, "conductas más masculinas". Raguz de Romaña, en una revisión de la literatura, señala que "las mujeres femeninas (alta femineidad/baja masculinidad autopercibidas) tienen menor logro en sus carreras"; y, agrega: "tanto en hombres como en mujeres, la autopercepción de su masculinidad -no así de su femineidad- predice efectivamente el éxito de sus carreras" (1988; pg. 93).

Revisemos seguidamente lo concerniente al PCTA. Para ello presentamos en la Tabla 6 los adjetivos en los cuales las diferencias tuvieron significación estadísticas, ordenados según el sexo que presenta el mayor promedio.

Lo que puede observarse en la Tabla 6 es que los adjetivos en los cuales los hombres puntúan más alto que las mujeres y tienen además una diferencia estadísticamente significativa son aquellos que mejor identifican el PCTA ("seguro", "ambicioso", "discutidor", "vigoroso"), mientras que los de las mujeres corresponden más a la imagen de una persona sociable. De ese modo, si bien las mujeres obtienen un promedio general más alto 202 
Tabla 6: Ítemes de mayor puntaje por sexo correspondientes a conductas tipo A y B

\begin{tabular}{ll}
\hline \multicolumn{1}{c}{ Mujeres } & Hombres \\
\hline Franco (A) & Seguro (A) \\
Emprendedor (A) & Rápido (A) \\
Tenso (A) & Ambicioso (A) \\
Entusiasta (A) & Dominante (A) \\
Sensible (B) & Discutidor (A) \\
Sereno (B) & Vigoroso (A) \\
Hablador (A) & \\
Sociable (A) & \\
\hline
\end{tabular}

son los hombres los que se revelan, en el análisis de cada uno de los ítemes, con mayor tendencia a la Conducta Tipo A.

Varias explicaciones se pueden dar para esto. Debe tenerse en cuenta que el PCTA es un concepto multidimensional (Eysenck \& Fulker, 1983), y que parece consistir en una combinación de neuroticismo y extraversión (Eysenck, 1991). Eysenck ha señalado asimismo que el PCTA "parece estar relacionado particularmente al ambiente laboral, mientras que el trabajo sobre estrés ha sugerido la importancia de las relaciones interpersonales, especialmente dentro de la familia" (p. 27). Dado que el Inventario de Blumenthal et al. posee sólo una validación estadística, puede ser que esté evaluando sobre todo algunos de los aspectos de la Conducta Tipo A. Los resultados del análisis factorial que reportamos en la Tabla 5 dan una idea de dichos aspectos y nos permiten afirmar que los hombres puntúan más alto en competitividad, egocentrismo y actividad, tres rasgos distintivos del PCTA; pero las mujeres están por encima de ellos en tensión, un elemento "tóxico" del PCTA. Seńalemos, por supuesto, que el Inventario de Blumenthal et al., de facto, está evaluando más actitudes que conductas, algo que han señalado Byrne et al. (1985) como una de las limitaciones de los cuestionarios de autoreporte.

Algunos aspectos de la investigación del PCTA en mujeres deben también ser considerados para la discusión. Lawler et al. (1991) han pasado 
revista a ella seńalando que los estudios con mujeres de colleges parecen sufrir de un sesgo de diferencias de género (gender differences bias) (HareMustin \& Marecek, 1988); esto es, que ellas representan "en general un grupo sano y con bajo riesgo para trastornos coronarios" (p. 141).

Esto da sustento a nuestra aseveración de que el hecho de trabajar con una muestra universitaria ha influido en los puntajes de las mujeres, en el sentido de que ellas presentan calificaciones más cercanas ( $y$ en algunos casos superiores) a los varones.

La información de Lawler et al. (1991) -junto con la información de la Tabla 5- posibilitaría una explicación al mayor puntaje total de las mujeres. Dado que el reactivo de Blumenthal et al. comprende varios factores, es probable que ciertos aspectos del PCTA no necesariamente vinculados con trastornos cardiaco-coronarios, como por ejemplo Conducta competitiva y Gregarismo (con nueve y cinco ítemes, respectivamente), hayan influido en el puntaje elevado de ellas. Los factores que nos parecen más ligados a dichos trastornos, Agresividad y Tensión sólo suman 7 ítemes, $y$, por ende, influyen menos en el puntaje final.

En todo caso, los resultados ofrecen material para posteriores discusiones. Una de ellas es la referida a la naturaleza psicométrica del reactivo con el cual hemos trabajado. Otra, es profundizar en el análisis de las diferencias por sexo considerando aspectos tales como la edad. No menos importante nos parece llevar a cabo nuevos estudios en los cuales se exploren y precisen las connotaciones de los términos empleados en el Blumenthal.

Pero quizás lo más importante sea la necesidad de llevar a cabo estudios en el área de la Psicología de la Salud con mujeres. Estas constituyen, como lo han señalado en trabajo reciente Rodin \& Ickovics (1990), un grupo que parece confrontar particulares riesgos en salud, dado que ciertas circunstancias médicas se producen exclusiva (o casi exclusivamente) en ellas (histeroctomías, cesáreas, dismenorreas, cáncer al seno) o en porcentajes elevados (artritis reumatoidea, lupus, osteoporosis). Por todo ello, trabajos en esta área son y serán de gran significado. 


\section{Referencias}

Acuña, L.; Bruner, C. A. \& Avila, R. (1994). Estructura factorial del Inventario de Roles Sexuales de Bem en México. Revista Interamericana de Psicologia, 28, 155-168.

Adler, N. \& Matthews, K. (1994). Health psychology: why do some people get sick and some stay well?. Annual Review of Psychology, 45, 229-259.

Amat y León, C. (1986). La desigualdad interior en el Perú. Lima: Centro de Investigación de la Universidad del Pacífico.

Anderson, J. (1987). Imágenes de la familia en los textos y vida escolares. Revista Peruana de Ciencias Sociales, 1, 115-135.

Baker, L. J.; Deaborn, M. J.; Hastings, J. \& Hamberger, K. (1984). Type

A behavior in women: a review. Health Psychology, 3, 447-497.

Banaji, M. \& Prentice, D. A. (1994). The self in social contexts. Annual Review of Psychology, 45, 297-322.

Barrig, M. (1988). Empleo y trabajo femenino. Revista Peruana de Ciencias Sociales, 1, 7-39.

Berman, M.; Gladue, B. \& Taylor, S. (1993). The effects of hormons, Type A behavior pattern, and provocation on aggression in men. Motivation and Emotion, 17, 125-138.

Best, D. L.; Williams, J. E.; Cloud, J. M. et al. (1977). Development of sex trait stereotypes among young children in the United States, England and Ireland. Child Development, 48, 1375-1384.

Blumenthal, J. A.; Herman, S.; O Toole, L. C.; Haney, Th. L.; Williams, Jr., R. B. \& Barefoot, J. C. (1985). Development of a brief self-report measure of the Type A (coronary prone) behaviour pattern. Journal of Psychosomatic Research, 29, 265-274.

Booth-Kewley, S. \& Friedman, H. J. (1987). Psychological predictors of heart disease: a quantitative review. Psychological Bulletin, 101, 343-362.

Byrne, D. G.; Rosenman, R. H.; Schiller, E. \& Chesney, M. A. (1985). Consistency and variation among instruments purporting to measure

Type A behavior partern. Psychosomatic Medicine, 47, 242-261.

Consoli, S. M. \& Antonelli, P. (1992). Profil comportamental de Type A, dépressivité et risque vasculaire. L'Encéphale, 18, 695-699.

Check, J. V. P: \& Dyck, D. G. (1986). Hostile aggression and Type A behavior. Personality and Individual Differences, 7, 819-827. 
Chueca, M. (1985). Sexualidad, fecundidad y familia en Villa El Salvador. En: Ponce, A.; Francke, M.; García, J. M.; Chueca, M. A. \& Chávez, E., Hogar y familia en el Perú, Lima, Pontificia Universidad Católica del Perú, 93-133.

Dembroski, Th. M. \& Czajkowski; S. M. (1989). Historical and current developments in coronary-prone behavior. En: Siegman, A. W. \& Dembroski, Th. M., eds., In search of coronary-prone behavior. beyond Type A, Londres: Lawrence Erlbaum, 21-39.

Eysenck, H. J. (1991). Type A behavior and coronary heart disease: the third stage. En: Strube, M. J., ed., Type A behavior, Londres: Sage, 25-44.

Eysenck, H. J. \& Fulker, D. (1983). The components of Type A behaviour and its genetic determinants. Personality and Individual Differences, 4, 499-505.

Feingold, A. (1988). Cognitive gender differences are disappearing. American Psychologist, 43, 95-101.

Ferrando, D.; Singh, S. \& Wulf, D. (1989). Adolescentes de hoy, padres del mañana: Peru. Nueva York: The Alan Guthmacher Institute.

Francke, M. (1986). Mujer, población y desarrollo. En: Guerra-García, R, ed., Problemas poblacionales peruanos II, Lima, Asociación Multidisciplinaria de Investigación y Docencia en Población, 299-352.

Friedman, M. \& Rosenman, R. H. (1959). Association of specific overt behavior pattern with blood and cardiovascular findings. Journal of the American Medical Association, 169, 1286-1296.

Frieze, I. H.; Parsons, J. E.; Johnson, P. B.; Ruble, D. N. \& Zellman, G. L. (1978). Women and sex roles. A social psychological perspective. Londres: W. W. Norton.

Furnham, A. (1990). The protestant work ethic. The psychology of work related beliefs and behaviours. Londres: Routledge.

García Trovato, M. (1992). Salud mental de la mujer en el Perú. En: Zambrano, M.; Gonzáles Norris, L. \& Castillo, A., eds., Psiquiatria Peruana/12. Anales del XII. Congreso Nacional de Psiquiatria, Lima, Biblioteca de Psiquiatría Peruana, 67-80.

Guzmán, V. \& Portocarrero, A. (1985). Dos veces mujer. Lima: Mosca Azul.

Hare-Mustin, R. T. \& Marecek, J. (1988). The meaning of difference: gender theory, postmodernism, and psychology. American Psychologist, 


\section{3, 455-464.}

Henley, S. \& Furnham A. (1989). The Type A behaviour pattern and selfevaluation. British Journal of Medical Psychology, 62, 51-59.

Holmes, D. S. \& Will, M. J. (1985). Expression of interpersonal aggression by angered and nonangered persons with the Type A and Type B behavior patterns. Journal of Personality and Social Psychology, 48, 723-727.

Holroyd, K. A. \& Coyne, J. (1987). Personality and health in the 1980s: psychosomatic medicine revisited?. Journal of Personality, 55, 359-375. Jose, P. E. \& McCarthy, W. J. (1988). Perceived agentic and communal behavior in mis-sex groups interactions. Personality and Social Psychology Bulletin, 14, 57-67.

Kemple, L. (1945). Rorschach method and psychosomatic diagnosis: personality traits of patients with rheumatic disease, hypertension, cardiovascular disease, occlusions and fracture. Psychosomatic Medicine, 7, 85-89.

Lawler, K. A.; Schmied, L. A.; Armstead, Ch. A. \& Lacy, J. E. (1991). Type A behavior, desire for control, and cardiovascular reactivity in young adult women. En: Strube, M. J., ed., Type A behavior, Londres: Sage, $135-158$.

Lazo Manrique, J. (1988). El adolescente en el Perú: investigación psicológica. Psicología y Sociedad, 1, 149-176.

León R. \& Portocarrero, C. (1989). Medición de la autoconciencia en adolescentes de una zona marginal en Lima (Perú). Avances en Psicologia Clinica Latinoamericana, 7, 39-54.

León, R. \& Romero, C. (1988). Conducta tipo A en un grupo de adolescentes de una zona deprivada de Lima Metropolitana: un estudio con el Inventario de Eysenck \& Fulker. Psicología y Sociedad, 1, 203-221.

León, R.; Romero, C. \& Sirlopú, D. (1995). Validación del Inventario de Autoreporte de Conducta Tipo A de Blumenthal et al. en un grupo de estudiantes universitarios de Lima Metropolitana. Revista de Neuropsiquiatría, 58 (1), en prensa.

León, R. \& Sirlopú, D. (1995). Factorial structure of the Type A SelfRating Inventory in a Peruvian sample. En: Die biopsychosoziale Einheit Mensch-Begegnungen. Festschrift für Prof. Dr. K. F. Wessel. Berlín: Universidad Humboldt.

Maccoby, E. E. (1988). Gender as a social category. Developmental Psychology, 24, 755-765. 
Maccoby, E. E. \& Jacklin, C. N. (1976). The psychology of sex differences. Stanford: Stanford University Press.

Majluf, A. (1989). Prácticas de crianza en madres de estratos socio-económico medio y baja de Lima. Revista de Psicología (PUCP), 7, 151161.

Matthews, K. A. \& Haynes, G. G. (1986). Type A behavior pattern and coronary disease risk. American Journal of Epidemiology, 123, 923-959.

McClelland, D. C. (1981). Child rearing versus ideology and social structure as factors in personality development. En: Munroe, R. H.; Munroe, R. L. \& Whiting, B. B., eds., Handbook of cross-cultural human development, Nueva York: Garland STOM Press, 73-90.

Menninger, R. A. \& Menninger, W. C. (1936). Psychosomatic observations in cardiac disorders. American Heart Journal, 11, 10-21. Osler, W. (1910). Lecture on angina pectoris. Lancet, 1, 839-844.

Perales, A.; Tejada, K.; Villanueva, M. \& Hagashi, S. (1985). Relación madre-hijo en una población urbano-marginal. Estudio piloto. Anales de Salud Mental, 1, 50-68.

Ragúz de Romaña, M. (1983). Estereotipos de rol sexual y diferencias sexuales: realidad y distorsión. Revista de Psicología (PUCP), 1, 27-37. Ragúz de Romaña, M. (1988). La mujer que trabaja: revisión de investigaciones sobre atribución de logro; preferencias y metas ocupacionales; actitudes hacia la mujer que trabaja. Revista de Psicología (PUCP), 6, 87- 101 .

Review Panel on Coronary-prone behavior and coronary heart disease (1981). Coronary-prone behavior and coronary heart disease: a critical review. Circulation, 63, 1199-1215.

Rodin, J. \& Ickcovics, J. R. (1990). Women's health. Review and research agenda as we approach the 21 st. century. American Psychologist, 45, 1018-1034.

Rosenman, R. H.; Brand, R. J.; Jenkins, C, D.; Friedman, M.; Straus, R. \& Wurm, M. (1975). Coronary heart disease in the Western Collaborative Group Study: final follow-up of 8 1/2 years. Journal of the American Medical Association, 233, 872-877.

Rosenman, R. H.; Brand, R. J.; Sholtz, R. I. \& Friedman, M. (1976). Multivariate prediction of coronary heart disease during 8.5 years follow-up in the Western Collaborative Group Study. American Journal of Cardiology, 37, 903-910.

Rosenman, R. H. Chesney, M. A. (1982). Stress, Type A behavior, and coronary disease. En: Goldenberg, L. \& Breznitz, Sh., eds., Handbook 
of stress. Theoretical and clinical aspects, Nueva York: The Free Press, 547-565.

Rosenman, R. H. \& Friedman, M. (1961). Association of specific behavior pattern in women with flood and cardiovascular findings. Journal of the American Medical Association, 24, 1173-1184.

Shoham-Yakubovich, I.; Ragland, D. R.; Brand, R. J. \& Syme, S. L. (1988). Type A behavior pattern and health status after 22 years of follow-up in the Western Collaborative Group Study. American Journal of Epidemiology, 128, 579-588.

Smith, T. W. \& Pope, M. K (1991). Cynical hostility as a health risk: current status and future directions. En: Strube, M. J., ed., Type $A$ behavior, Londres: Sage, 117-133.

Spence, J. T. \& Helmreich, R. L. (1978). Masculinity and femineity. Their psychological discussions, correlates and antecedents. Austin: University of Texas Press.

Stoller, R. J. (1981). Identidad del género. En: Krauss, St., dir. (1981). Enciclopedia de psicología médica, Barcelona, El Ateneo, 274-276.

Strube, M. J.; Turner, C. W.; Cerro, C.; Stevens, J. \& Hinchey, F. (1984). Interpersonal aggression and the Type A coronary-prone behavior pattern: a theoretical distinction and practical implications. Journal of Personality and Social Psychology, 47, 839-847.

Thoresen, C. E. \& Low, K. G. (1991). Women and the Type A behavior pattern: review and commentary. En: Strube, M. J., ed., Type $A$ behavior, Londres: Sage, 117-133.

Tyler, L. (1972). Psicología de las diferencias humanas. Madrid: Marova. Williams, J. E.; Benett, S. M. \& Best, D. L. (1975). Awareness and expression of sex stereotypes in young children. Developmental Psychology, 11, 635-642.

Williams, R. B.; Lane, J. D.; Kuhn, C. M.; Melosh, W.; White, A. D. \& Schanberg, S. M. (1982). Type A behavior and elevated physiological and neuroendocrine responses to cognitive tasks. Science, 218, 483-485. 